\title{
硫代草酸 $-(\mathbf{O}, \mathbf{S})$ 二(乙二胺 $)$ 合钴 (III) 配合物的光化学*
}

\author{
陈 忠 张静智 丁世良 沈晋明 \\ (山东大学化学系, 济南)
}

\section{一、引 妄}

近年来, $\mathrm{Co}(\mathrm{en})_{2} \mathrm{~L}^{\mathrm{n}+}(\mathrm{en}$ 是乙二胺, $\mathrm{L}$ 表示含一个配位原子硫的整合配体) 类型的 $\mathrm{Co}(\mathrm{III})$ 篮合配合物已成为广泛研究的课题, 关于它们的合成、结构及热反应已有了许多的近期 报 道 ${ }^{[1,2]}$. Adamson 等研究了一系列含硫和硒的这类 $\mathrm{Co}(\mathrm{III})$ 瞥合配合物的光化学, 并把这些配 合物分成三类 ${ }^{[3]}$.

我们对含有硫代草酸盐的 $\mathrm{Co}(e n)_{2} \mathrm{~L}^{\mathrm{n}}$ 类型的 $\mathrm{Co}(\mathrm{III})$ 慜合配合物的光化学感到兴趣, 因 为含有一个配位原子硫的这类配合物的光化学报道很少, 而且在硫代草酸盐配体的这种配合 物中, 有硫与氧原子与 $\mathrm{Co}$ (III) 中心配位 ${ }^{[4]}$. 在我们的研究中, 我们能把含 $\mathrm{Co}-\mathrm{S}$ 键的 $\mathrm{Co}$ (III) 配合物的光化学与含 $\mathrm{Co}-\mathrm{O}$ 键的 $\mathrm{Co}(\mathrm{en})_{2}(\mathrm{OX})^{1+}$ ( $\mathrm{OX}$ 是草酸根) 类型的光化学进行 比较. 因为 $\mathrm{Co}(\mathrm{en})_{2}(\mathrm{OX})^{1+}$ 配合物在用紫外光照射时, 发生还原分解 ${ }^{[3]}$, 这样, 我们就能比较 含 $\mathrm{Co}-\mathrm{S}$ 键和 $\mathrm{Co}-\mathrm{O}$ 键的配合物对光的稳定性.

\section{二、实验}

1. 材料实验中使用的化学药品均是化学纯试剂, 合成硫代草酸钾所用的试剂乙醇先 用金属钾干燥, 再蒸馏.

2. 仪器 HP8451A UV-Vis 分光光度计及 Oriel 200 瓦 $\mathrm{Hg}-\mathrm{Xe}$ 弧灯.

3. 合成硫代草酸钾的合成, 采用文献方法 ${ }^{[6]}$, 但略加改进. 使用 $2 \mathrm{~mol} / 1$ 的粗制配体 同 $1 \mathrm{~mol} / 1 \mathrm{CaCl}_{2}$ 反应, 并过滤去草酸钻, 来除去混在硫代草酸盐中的杂质草酸钾. 元素分析 及红外光谱表明, 产品具有较好的纯度. cis- $\left[\mathrm{Co}(\mathrm{en})_{2} \mathrm{Cl}_{2}\right] \mathrm{Cl}^{[7]}$ 及 $\left[\mathrm{Co}(\mathrm{en})_{2}\left(\mathrm{SC}_{2} \mathrm{O}_{3}\right)\right] \mathrm{Cl}$. $\mathrm{H}_{2} \mathrm{O}^{[4]}$ 均按文献方法合成. $\left[\mathrm{Co}(\mathrm{en})_{2}\left(\mathrm{SC}_{2} \mathrm{O}_{3}\right)\right] \mathrm{Cl} \cdot \mathrm{H}_{2} \mathrm{O}$ 在高氯酸桸溶液中的吸收光谱, $512 \mathrm{~nm}$ 处的摩尔消光系数 $\varepsilon=155.6 \mathrm{M}^{-1} \mathrm{~cm}^{-1}$, 与文献值 $156 \mathrm{M}^{-1} \mathrm{~cm}^{-1}(512 \mathrm{~nm})$ 基本相符.

4. 光分解 $\mathrm{Hg}-\mathrm{Xe}$ 弧灯的白光经 $\mathrm{CoSO}_{4}$ 和 $\mathrm{NiSO}_{4}$ 溶液滤光以取得宽带的近 外 光 ${ }^{[8]}$. 溶液滤光池为 $75 \times 35(\mathrm{~mm})$ 的圆柱形石英管, 其周围有流水冷却. 它们在紫外区的最 大发射波长分别为 $362 \mathrm{~nm}$ 和 $310 \mathrm{~nm}$, 典型人射光强度分别是 $1.91 \times 10^{-4}$ einstein $\mathrm{L}^{-1} \mathrm{~S}^{-1}$ 和 $1.28 \times 10^{-4}$ einstein $\mathrm{L}^{-1} \mathrm{~S}^{-1}$. 光强度测定使用草酸铁钾化学光量计 ${ }^{[9]}$, 在光分解前后各进行一 次测定, 取其平均值计算量子效率. 光分解是在 $1 \mathrm{~cm}^{2}$ 的带塞石英比色池中进行的, 使用的配 合物浓度为 $1.44 \times 10^{-3} \mathrm{~mol} / \mathrm{l}$, 它对照射光有完全的吸收. 在 $0.01 \mathrm{~mol} / 1 \mathrm{HClO}$ ，和水中光照

- 国家目然科学基金资助项目. 
时, 通人氮气泡进行摚拌, 在 $12 \mathrm{~mol} / 1 \mathrm{HCl}$ 中光照时, 则盖上比色池的磨口塞.

5. 动力学测定 在 $12 \mathrm{~mol} / 1 \mathrm{HCl}$ 的 $1.44 \times 10^{-3} \mathrm{~mol} / 1$ 配合物溶液, 分别用 CoSO, 和 $\mathrm{NiSO}$ 、溶洨滤光得到的宽带近紫外光照射, 反应程度可以通过变化最大的 $690 \mathrm{~nm}$ 处的吸收来 观察 (如图 1). 以 $\ln \left(D_{\infty}-D_{t}\right)$ 对 $t$ 作图, 在照射时间范围内是一条直线, 用最小二乘法计算 准一级速度常数 $K_{p}$.

6. 产物分析和年子效率 在 $12 \mathrm{~mol} / 1 \mathrm{HCl}$ 中的量子效率 $\phi_{\mathrm{Co}^{2}}{ }^{2}$ 是从 $\mathrm{Co}$ (II) 的浓度对 照射时间图的最初斜率来计算的. $\mathrm{Co}$ (II) 浓度的数值是用分光光度法测定光分解溶液 在 不 同照射时间的吸收来计算的, 使用的蓝色氯化钴 (II) $\mathrm{CoCl}_{4}^{2-}$ 在 $690 \mathrm{~nm}$ 的克分子消光系数 $\varepsilon=548.4 \mathrm{M}^{-1} \mathrm{~cm}^{-1[10]}$. 对其他的光分解溶液,是将一定量的光照溶液溶在 $12 \mathrm{~mol} / 1 \mathrm{HCl}$ 中, 用 分光光度法, 测定在 $690 \mathrm{~nm}$ 的吸收, 来计算形成的 (II)的浓度. 草酸根离子的检测 采用文 献的方法 ${ }^{[1]}$. 为了确定原来配位在硫代草酸 $-(O, S)$ 二(乙二胺)合钴 (III) 分子中的乙二胺 分解成氨,还是在照射后,乙二胺从钴上脱离,但未分解,我们做了下面的实验: 将几毫升光照 液或纯乙二胺放在表面皿上, 加入氢堇化钾溶液, 并分别在水浴上加热, 它们均能使试纸变蓝, 但在光解液中加入奈氏试剂, 却无氨的特征反应, 说明从 Co(III) 上脱下的乙二胺, 在光照时 并未分解.

\section{三、结果}

1. 在 $12 \mathrm{~mol} / 1 \mathrm{HCl}$ 中的光照反应在 $12 \mathrm{~mol} / 1 \mathrm{HCl}$ 中的 $1.44 \times 10^{-3} \mathrm{~mol} / 1$ 配合物溶液, 分别经 $\mathrm{CoSO}_{4}$ 和 $\mathrm{NiSO}_{4}$ 滤光液的近紫外光照射, 照射溶液的吸收光谱在 $690 \mathrm{~nm}$ 处增加, 证明 形成了蓝色的 $\mathrm{CoCl}_{4}^{2-}$. 图 1 说明该配合物在 $12 \mathrm{~mol} / 1 \mathrm{HCl}$ 中用 $\mathrm{NiSO}_{4}$ 滤光液的光照时, 吸收 光谱的变化. 光琞初期, 在 450-600 $\mathrm{nm}$ 范围内有两个等吸收点, 该溶液照射 180 秒后, 在 400-500 nm 范围，吸收明显增加. 长时间照射后，可观察到照射溶液中有硫析出. 根据在 $690 \mathrm{~nm}$ 吸收的增加, 以 $\ln \left(D_{\infty}-D_{t}\right)$ 对 $\mathrm{t}$ 作图, 在一定照射时间内为一直线, 直线的斜率是 准一级速度常数 $K_{p}$. 图 2 说明配合物在 $12 \mathrm{~mol} / 1 \mathrm{HCl}$ 中的准一级反应图. 经 CoSO، 滤光液的 光照, 吸收光强 $I_{a}=1.91 \times 10^{-4}$ einstein $1^{-1} \mathrm{~s}^{-1}, K_{p}=(6.36 \pm 0.03) \times 10^{-3} \mathrm{~s}^{-1}$; 而经 NiSO

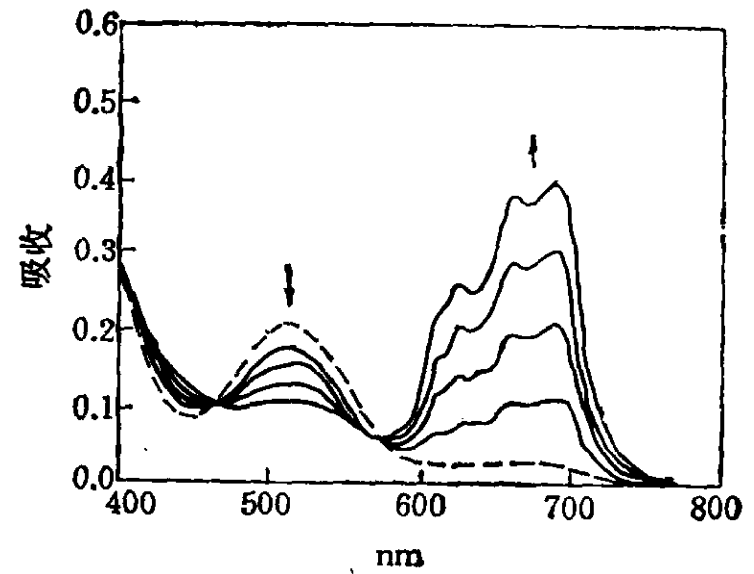

图 1 在 $12 \mathrm{~mol} / 1 \mathrm{HCl}$ 中的 $1.44 \times 10^{-3} \mathrm{~mol} / 1$ 配合物, $25^{\circ} \mathrm{C}$ 用 $\mathrm{NiSO}_{4}$ 溶液䍇光进行光解时的吸收光谱变化 应线为炤射前的光谱，依次向上分别为光炤 $30,60,90,120 \mathrm{~s}$

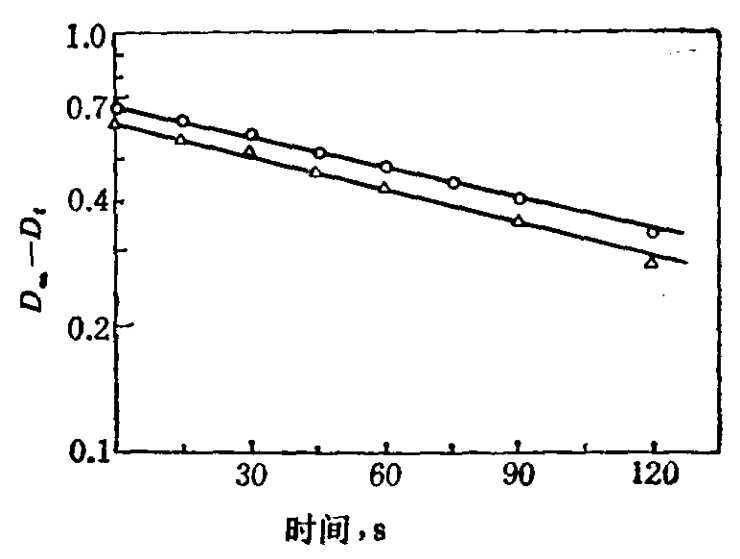

图 2 在 $12 \mathrm{~mol} / 1 \mathrm{HCl}$ 中, $25^{\circ} \mathrm{C}$ 的准一级反应图 $0-\mathrm{NiSO}_{4}$ 溶液波光 $l_{\mathrm{s}}=1.28 \times 10^{-4} \mathrm{ein} 8 \mathrm{tein} \mathrm{L}^{-1} \mathrm{~g}^{-1}$,

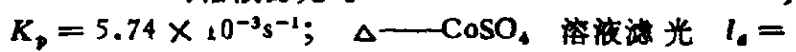
$1.91 \times 10^{-4}$ einstein $\mathrm{L}^{-1} \mathrm{~S}^{-1} \mathrm{~K}_{\mathrm{p}}=6.32 \times 10^{-3} \mathrm{~s}-1$ 
滤光液的光照, $I_{a}=1.28 \times 10^{-4}$ einstein $1^{-1} \mathrm{~s}^{-1}, K_{p}=(5.76 \pm 0.02) \times 10^{-3} \mathrm{~s}^{-1}$.

在不同照射时间, 测定 $\mathrm{Co}$ (II) 的浓度, 并以 $\mathrm{Co}$ (II) 的浓度对时间作图，在一定照射时间 内为一直线 (图 3). 对 $\mathrm{CoSO}_{4}$ 和 $\mathrm{NiSO}_{4}$ 溶液滤光, $\mathrm{Co}$ (II) 生成的速度分别为 $(5.29 \pm 0.10) \times 10^{-6} \mathrm{~mol} / 1$ $\mathrm{s}^{-1}$ 和 $(5.25 \pm 0.23) \times 10^{-6} \mathrm{~mol} / 1 \mathrm{~s}^{-1}$, 从图 3 的斜率 计算的量子效率 $\phi \mathrm{C}_{0}{ }^{2+}$ 分别为 $(0.029 \pm 0.001) \mathrm{mol}$ einstein $^{-1}$ 和 $(0.042 \pm 0.002) \mathrm{mol}$ einstein ${ }^{-1}$.

\section{2. 在 $0.01 \mathrm{~mol} / 1 \mathrm{HClO}$ 、溶液中的光照反应}

该配合物在 $0.01 \mathrm{~mol} / 1 \mathrm{HClO}_{4}$ 溶液中是稳定的, 即使 放置两周或加热至沸，也未观察到该溶液吸收光谱的 变化. 当配合物的 $1.44 \times 10^{-3} \mathrm{~mol} / 1$ 的溶液用宽带的 近紫外光照射, 红色溶液渐渐祖去, 成无色透明, 光照 $100 \mathrm{~s}$ 以上, 出现孚白色硫沉淀. 图 4 为在 $0.01 \mathrm{~mol} / 1$ $\mathrm{HClO}_{4}$ 的 $1.44 \times 10^{-3} \mathrm{~mol} / 1$ 配合物, 用 $\mathrm{CoSO}_{4}$ 滤光液 的光炤射时, 吸收光谱的变化. 光照初期, 在 $512 \mathrm{~nm}$ 的吸收减小，照射 60 s 后，吸收增加．特别在 400$450 \mathrm{~nm}$ 范围内更为明显, 并观察到乳白色沉淀, 这是由 于形成胶体硫的原因. 经 $\mathrm{CoSO}_{4}$ 和 $\mathrm{NiSO}_{4}$ 溶液滤光 的量子效率 $\phi_{\mathrm{Co}^{2}}{ }^{2}$ 分别为 $(0.111 \pm 0.001) \mathrm{mol}$ einstein ${ }^{-1}$ 和 $(0.124 \pm 0.001) \mathrm{mol}$ einstein ${ }^{-1}$.

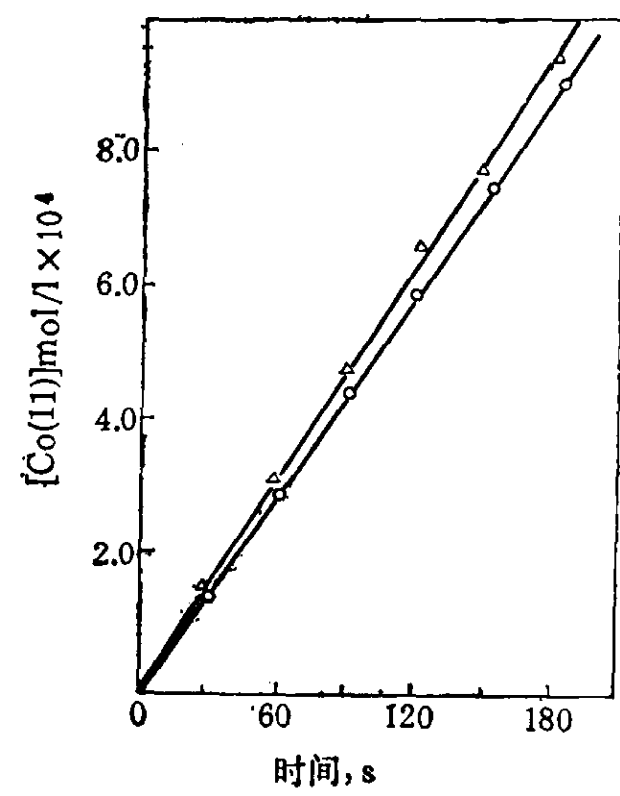

图 3 在 $25^{\circ} \mathrm{C} \mathrm{Co}$ (II) 的浓度随炤 射时间的变化

O- NiSO 4 溶液浡光 $l_{a}=1.28 \times 10^{-4}$ einst ein $1^{-1} 8^{-1}, S=5.02 \times 10^{-6} \mathrm{~mol} / 1 \mathrm{~s}^{-1} ; \Delta-$ $\mathrm{CoSO}_{4}$ 溶液㴓光 $I_{a}=1.91 \times 10^{-4}$ einstein $1^{-1}$ $\mathrm{s}^{-1}, S=5.19 \times 10^{-6} \mathrm{~mol} / 1 \mathrm{~s}^{-1}$

3. 在水中的光照反应在水中的 $1.44 \times 10^{-3} \mathrm{~mol} / \mathrm{l}$ 配合物溶液, 经 $\mathrm{CoSO}_{4}$ 和 $\mathrm{NiSO}$ 、 滤光液的光照射, 它们的吸收光谱变化相同. 图 5 为经过 $\mathrm{CoSO}$ 滤光液的光照, 配合物吸收光 谱的变化. 由图可见, 吸收曲线逐渐升高, 配合物溶液的颜色由红变为棕黑色, 产生了 $\operatorname{CoS}$ 沉 淀. 将光照液过滤, 滤液的 $\mathrm{pH}$ 值从照射前的 7.8下降到 7.5, 这是因为在光照时产生硫化 氢的原因. 经 $\mathrm{CoSO}_{4}$ 和 $\mathrm{NiSO}_{4}$ 滤光液的光照, 测得的量子效率 $\phi_{\mathrm{Co}^{2}+}$ 分别为 $(0.051 \pm 0.001)$

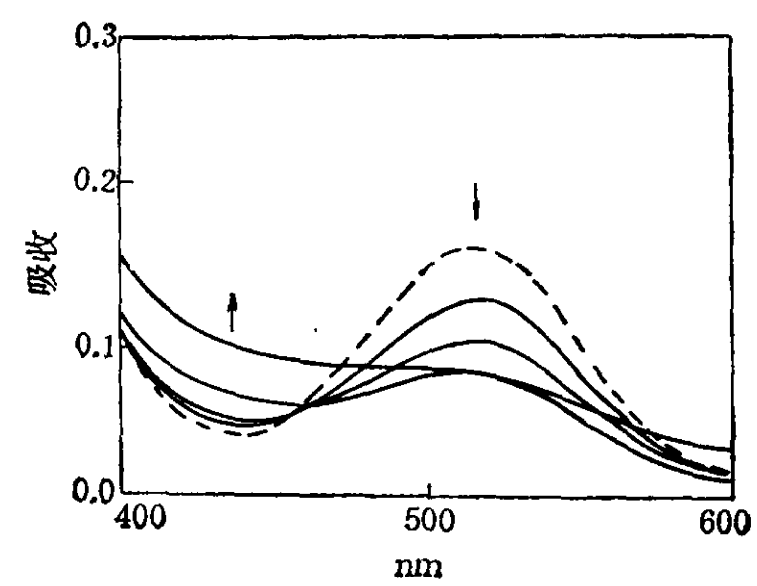

图 4 在 $0.01 \mathrm{~mol} / 1 \mathrm{HClO}_{4}$ 的 $1.44 \times 10^{-3} \mathrm{~mol} / 1$ 配化 物用 $\mathrm{CoSO}$ 、流光液的光照射,吸收光谱的变化 线为光用前，实线由上而下分别光盟 $15,30 ， 45,60 \mathrm{~s}$

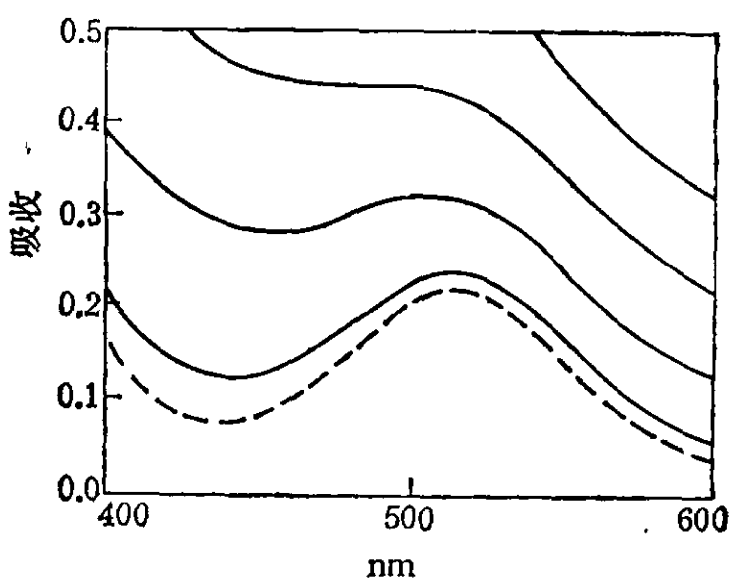

图 5 在水中 $1.44 \times 10^{-3} \mathrm{~mol} / 1$ 配合物用

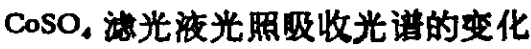


表 $1 \mathrm{Co}$ (II) 生成的量子效率

\begin{tabular}{|c|c|c|}
\hline $\begin{array}{c}\text { 盟射波长 } \\
\text { UV }\end{array}$ & 塎 & $\begin{array}{c}\Phi_{\mathrm{Co}^{2+}} \\
\text { mol einstein }\end{array}$ \\
\hline CoSO 。溶液滤光 & $\begin{array}{l}12 \mathrm{~mol} / 1 \mathrm{HCl} \\
0.01 \mathrm{~mol} / 1 \mathrm{HClO} \text {, } \\
\text { 水 }\end{array}$ & $\begin{array}{l}0.029 \pm 0.001 \\
0.111 \pm 0.001 \\
0.051 \pm 0.001\end{array}$ \\
\hline NisO，溶波潗光 & $\begin{array}{l}12 \mathrm{~mol} / 1 \mathrm{HCl} \\
0.01 \mathrm{~mol} / 1 \mathrm{HClO} \text {, } \\
\text { 水 }\end{array}$ & $\begin{array}{l}0.042 \pm 0.002 \\
0.124 \pm 0.001 \\
0.091 \pm 0.001\end{array}$ \\
\hline
\end{tabular}

mol einstein ${ }^{-1}$ 和 $(0.091 \pm 0.001) \mathrm{mol} \mathrm{einstein}^{-1}$.

在 $12 \mathrm{~mol} / 1 \mathrm{HCl}, 0.01 \mathrm{~mol} / 1 \mathrm{HClO}_{4}$ 和水中, $\mathrm{Co}$ (II) 生成的量子效率列于表 1 .

\section{四、讨 论}

该配合物在稀高氯酸水溶液中,有三个吸收峰, 分别在 $512 \mathrm{~nm}\left(8156 \mathrm{~mol}^{-1} \mathrm{~cm}^{-1}\right), 273 \mathrm{~nm}$ (8 $15600 \mathrm{~mol}^{-1} \mathrm{~cm}^{-1}$ ) 和 $215 \mathrm{~nm}\left(\varepsilon 16100 \mathrm{~mol}^{-1} \mathrm{~cm}^{-1}\right.$ ). 显然, 第一个吸收带是配位场类 型, 后两个吸收具有较高消光系数, 因而认为是从配体到金属离子的电子荷移带. 在配位场昭 射, 因为配合物对可见光稳定, 未发生配合物的光还原. 用宽带的近紫外光炤射, 电子荷移带 的吸收造成一个电子从硫向钴中心离子传递，而发生氧化还原反应. 因为该配合物对可见光 沅作用, 并且在这两个滤光液的可见区、配合物的消光系数很小, 因此在光分解实验中仍保留 着 $\mathrm{CoSO}_{4}$ 和 $\mathrm{NiSO}_{4}$ 滤光液的可见光部分. 对不同的光分解溶液,均能观察到形成 $\mathrm{Co}$ (II). 在 水中光分解产生的 $\operatorname{CoS}$ 沉淀滤去后, 从滤液中能检查出草酸根离子, 并且它的吸收光谱与碱 性水解滤液的吸收光谱相同. 文献已报道, 该配合物经过快速的碱性水解, 产生 $\mathrm{H}_{2} \mathrm{~S}, \mathrm{Co}$ (II) 和游离的草酸根 ${ }^{[4]}$. 当光照时, 配合物在水中生成 $\mathrm{CoS}$ 沉淀, 而在 $12 \mathrm{~mol} / 1 \mathrm{HCl}$ 中产生蓝色 的 $\mathrm{CoCl}_{4}^{-}$.

当用宽带的近紫外光照射时，激发态分子会发生电子从配位原子硫到钴中心的电子传递， 并造成 $\mathrm{Co}-\mathrm{S}$ 键破裂. 在 $0.01 \mathrm{~mol} / 1 \mathrm{HClO}$ 和 $12 \mathrm{~mol} / 1 \mathrm{HCl}$ 中, 经光炤观察到硫的沉淀, 就 是该光反应机制的证明. 根据这些结果,我们建议整个的反应为

$$
2 \mathrm{Co}(\mathrm{en})_{2}\left(\mathrm{SC}_{2} \mathrm{O}_{3}\right)^{1+}+2 \mathrm{H}_{2} \mathrm{O} \stackrel{h \nu}{\longrightarrow} \mathrm{H}_{2} \mathrm{~S}+\mathrm{S}+2 \mathrm{H}^{+}+2 \mathrm{Co}^{2+}+4 \mathrm{en}+2 \mathrm{C}_{2} \mathrm{O}_{4}^{2-} \text {. }
$$

该反应的步骤是 $(*=$ 激发态, $\Delta-$ 释放的热):

(1) 能量吸收

$$
\mathrm{Co}(\mathrm{en})_{2}\left(\mathrm{SC}_{2} \mathrm{O}_{3}\right)^{1+}+h \nu \stackrel{\mathrm{K}_{3}}{\longrightarrow} \mathrm{Co}^{*}(\mathrm{en})_{2}\left(\mathrm{SC}_{2} \mathrm{O}_{3}\right)^{1+},
$$

（2）能量消失

$$
\mathrm{Co}^{*}(\mathrm{en})_{2}\left(\mathrm{SC}_{2} \mathrm{O}_{3}\right)^{1+} \stackrel{K_{-1}}{\longrightarrow} \mathrm{Co}(\mathrm{en})_{2}\left(\mathrm{SC}_{2} \mathrm{O}_{3}\right)^{1+}+\Delta,
$$

（3）键破裂及电子传递

$$
2 \mathrm{Co}^{*}(\mathrm{en})_{2}\left(\mathrm{SC}_{2} \mathrm{O}_{3}\right)^{1+}+2 \mathrm{H}_{2} \mathrm{O} \stackrel{K_{2}}{\longrightarrow} \mathrm{H}_{2} \mathrm{~S}+\mathrm{S}+2 \mathrm{H}^{+}+2 \mathrm{Co}^{2+}+4 \mathrm{en}+2 \mathrm{C}_{2} \mathrm{O}_{4}^{2-} \text {. }
$$

令 $A, B, C$ 分别表示反应物, 激发态和产物 $\mathrm{Co}$ (II), $I_{4}$ 是反应物吸收光的强度, 我们就能 很容易得到下面的方程。 


$$
\begin{aligned}
& \stackrel{K_{1}, I_{a}}{\longrightarrow} \mathrm{B} \stackrel{K_{2}}{\longrightarrow} \mathrm{C}, \\
& \frac{d[\mathrm{~A}]}{d t}=-K_{1} I_{a}+K_{-1}[\mathrm{~B}], \\
& \frac{d[\mathrm{~B}]}{d t}=K_{1} I_{a}-K_{-1}[\mathrm{~B}]-K_{2}[\mathrm{~B}], \\
& \frac{d[\mathrm{C}]}{d t}=K_{2}[\mathrm{~B}],
\end{aligned}
$$

当 $t=0,[\mathrm{~A}]=\left[\mathrm{A}_{0}\right],[\mathrm{B}]=[\mathrm{C}]=0$.

通常假设激发态的浓度很快达到了一个固定值, 我们得到 ${ }^{[12]}$

$$
\begin{aligned}
& K_{1} I_{a}-K_{-1}[\mathrm{~B}]-K_{2}[\mathrm{~B}]=0, \\
& {[\mathrm{~B}]=\frac{K_{1}}{K_{-1}+K_{2}} I_{a} .}
\end{aligned}
$$

将方程 (8) 代人方程 (5) 和 (7), 再对时间 $\bullet$ 积分, 我们得到下面的表达式

$$
\begin{aligned}
{[\mathrm{A}] } & =\left[\mathrm{A}_{0}\right]-K_{1} I_{a} t+\frac{K_{-1} K_{1}}{K_{-1}+K_{2}} I_{a} t \\
& =\left[\mathrm{A}_{0}\right]-\frac{K_{1} K_{2}}{K_{-1}+K_{2}} I_{a} t, \\
{[\mathrm{C}] } & =\frac{K_{1} K_{2}}{K_{-1}+K_{2}} I_{a} t .
\end{aligned}
$$

随着照射时间，反应物的浓度线性减少，而产物的浓度线性增加. 直线的斜率是

$$
\frac{K_{1} K_{2}}{K_{-1}+K_{2}} I_{a}
$$

对 $\mathrm{CoSO}_{4}$ 和 $\mathrm{NiSO}_{4}$ 溶液滤光, 分别为 $(5.29 \pm 0.10) \times 10^{-6} \mathrm{~mol} / 1 \mathrm{~s}^{-1}$ 和 $(5.25 \pm 0.23) \times 10^{-6}$.6 $\mathrm{mol} / 1 \mathrm{~s}^{-1}$, 如图 3 所示.

致谢: 我们感谢邓从豪教授的帮助和指导. 颜奾华参加了部分研究.

\section{\% 文 塥}

[1] Elder, R. C. et al., Inorg. Chem., 17(1978), 431.

[2] Sloan, C. P. \& Krueger, J. H., Inorg. Chem, 14(1975), 1481.

[3] Houlding, V. H., Mäcke, H. \& Adamson, A. W., Inorg. Chem, 20(1981), 4279.

[4] Dlydon, J. D. et al., Inorg. Chem., 19(1980), 2083.

[ 5 ] Endicott, J. F., Hoffman, M. Z. \& Mollicone, B. L., 152nd A. C. S. Meering, Abstract of Papers, V. 38, New York. 1966.

[ 6 ] Leitheiser, M. \& Coucouvanis, D., J. Inorg. Nucl. Chem, 39(1977), 811.

[ 7 ] Bailar, J. C., Inorg. Synzh., 2(1946), 223.

[ 8 ] Calvert, J. C. \& Pitts, J. N., Photochemistry., Wiley, New York, 1966.

[9] Hatchard, G. C. \& Parker, C. A., Proc. R. Soc, London. Ser. A, 235(1956), 518.

[10] Endicott, J. F. \& Hoffman, M. Z., J. Am. Chem. Soc, 87(1965), 3348.

[11] Fernando Burriel-Marti et al., Anal. Chem, 25(1953), 583.

[1:] Balzani, V: Carassiti, V., Photochemistry of Coordination Compounds, Academic, New York, 1970, 9. 